\title{
Use of piezoelement in ultrasound generation
}

\author{
Shakhnoza Sultanova ${ }^{1 *}$, Jasur Safarov ${ }^{1}$, Azamat Usenov $^{1}$, Kamola Samatova ${ }^{1}$ \\ ${ }^{1}$ Tashkent State Technical University, Tashkent, Uzbekistan
}

\begin{abstract}
At present, ultrasonic (piezoelectric) transducers are the most promising electromechanical transducers for use in miniature electronic devices. However, the calculation of the piezoelectric element for an ultrasonic environment poses certain problems due to the large number of problems to be solved and its nonlinearity. The article describes the principles of operation of the piezoelectric element, analyzes their advantages and disadvantages, gives the characteristics of the piezoelectric elements, as well as proposes an algorithm for calculating the frequency characteristics of the piezoelectric element based on the electrical model of the piezoelectric element.
\end{abstract}

Key words: piezoelectric element, ultrasonic.

\section{Introduction}

In 1986 [1], for the first time on the pages of scientific periodicals, the abbreviation microelectromechanical structures (MEMS) appeared, which replaced the long and long pronounced phrase MEMS. Currently, the leading manufacturers of radio electronic components serially produce a fairly extensive list of elements, which include various MEMS. These are, first of all, various accelerometers produced in multi-million editions, resonators and filters of electrical signals realized on their basis, transformers and other microminiature electromechanical systems. MEMS production technologies are now called microsystem technologies.

MEMS or, equivalently, piezoelectric elements made using microsystem technologies have much in common with conventional, that is, non-microscopic, piezoelectric piezoelectric elements. To implement certain functions, MEMS uses polycrystalline ferroelectrics polarized by a constant electric field in a given direction. Conventional piezoelectric elements are made from piezoelectric ceramics, which is originally a polycrystalline ferroelectric polarized by a constant electric field of a given orientation at the last technological stage of manufacturing a piezoceramic product. A distinctive feature of MEMS from conventional piezoelements is the method of electroplating the working surfaces. Conventional piezoelectric cells usually have a solid galvanic surface. In some special cases, the electrodes are divided (cut) into separate sections that are not galvanically connected to each other. In MEMS, as a rule, partial electroplating of working surfaces is used, when only a part of the surface of a polarized ferroelectric is covered with a

\footnotetext{
${ }^{*}$ Corresponding author

E-mail: sh.sultanova@yahoo.com
}

metal film. This method of electroplating makes it possible to excite several types of elastic vibrations in the MEMS volume. By manipulating the geometric parameters of galvanic surfaces, it is possible to control the energetics of oscillatory processes in MEMS, that is, to create conditions when one type of oscillatory motion will prevail over the rest in terms of the amplitude of the vector of elastic displacements of material particles.

The piezoelectric effect is widely used today in various electromechanical devices and systems. Also widely used in ultrasonic generation. Therefore, the predictability of the characteristics of piezoelectric elements in the production of such systems is the main design task. The proposed algorithm for calculating the frequency characteristics of a piezoelectric element allows, on the basis of data provided by a piezoceramic manufacturer, to determine such parameters of a piezoelectric element of a given shape as its resonant and antiresonant frequencies, passband and cutoff frequencies. The calculation of these parameters is simple, with its help it is possible to construct a family of frequency characteristics for various sizes of a piezoelectric element, which allows developers of electromechanical systems based on the piezoelectric effect to determine the optimal design of a piezoelectric element, a sensitive or triggered element.

\section{Studying the piezoelectric effect}

In the absence of external mechanical stresses, the dipole moment of the unit cell is zero (Fig. 1). If, under the action of such stresses, the cell is stretched or compressed, then an electric dipole moment arises. Will be equal: $\mathrm{P}= \pm \mathrm{q} \cdot \Delta \alpha, \quad \mathrm{q}-$ ion charge, $\Delta \alpha-$ stretching or shrinking a cell [2-6].

The presence of electric dipole moments inside the piezoelectric element is detected by the appearance of electric 
charges on the opposite faces of the crystal or on the electrodes of the piezoelectric element (Fig. 1).
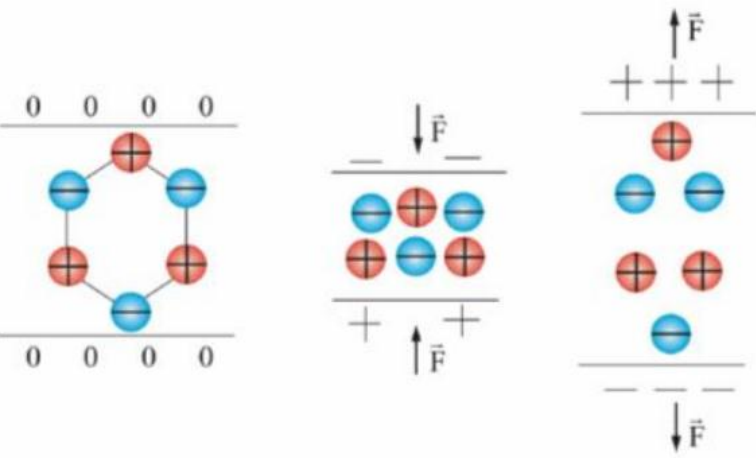

Fig. 1. Piezoelectric effect formation diagram.

The magnitude of the charge resulting from the piezoelectric effect is determined by the relationship:

$$
q=d_{i j} F_{x}
$$

where, $F_{x}$ - amount of force causing deformation; $d_{i j}-$ piezomodule (generally a tensor).

The reverse piezoelectric effect should not be confused with the phenomenon of electrostriction, that is, with the deformation of a dielectric under the influence of an electric field. With electrostriction, there is a quadratic relationship between the deformation and the field, and with the piezoelectric effect, it is linear. In addition, electrostriction occurs in a dielectric of any structure and even in liquids and gases, and the piezoelectric effect is observed only in solid dielectrics, mainly crystalline ones.

Piezoelectricity occurs only in those cases when the elastic deformation of the crystal is accompanied by a displacement of the centers of the location of positive and negative charges of the unit cell of the crystal, that is, it causes an individual dipole moment necessary for the occurrence of electric polarization of the dielectric under the action of mechanical stress. In structures with a center of symmetry, no uniform deformation can disturb the internal equilibrium of the crystal lattice, and, therefore, crystals of only those classes that do not have a center of symmetry are piezoelectric. The absence of a center of symmetry is a necessary but not sufficient condition for the existence of the piezoelectric effect; therefore, not all acentric crystals possess it. The piezoelectric effect cannot be observed in solid amorphous and cryptocrystalline dielectrics (almost isotropic), since this contradicts their spherical symmetry. The exceptions are cases when they become anisotropic under the action of external forces and thereby partially acquire the properties of single crystals [7-9].

\section{Characteristics of natural and artificial piezo materials}

Natural and artificial piezo crystals and piezo ceramics are currently one of the most important materials used in ultrasonic and sonic technologies. Table 1 shows the main characteristics of a number of piezoelectric materials.
Quartz. Due to its high temperature resistance and mechanical strength, quartz is currently used in laboratory and industrial practice. It does not dissolve in water and acids, is refractory (melting point $1470{ }^{\circ} \mathrm{C}$ ), loses its piezoelectric properties at a temperature of $570{ }^{\circ} \mathrm{C}$. Up to this temperature, quartz has a stable piezoelectric effect and can work effectively at high temperatures up to $500{ }^{\circ} \mathrm{C}$. A quartz crystal is shaped like a hexahedral prism with pyramid-like tapering at the ends [9-16]. The $\mathrm{z}$ axis, which runs parallel to the prism faces through the vertices of the opposite ends, is the optical axis of the crystal. The line passing through opposite corners of the prism perpendicular to the optical zaxis is the crystal's electric axis or the x-axis. The y-axis is directed perpendicularly to the opposite faces of the prism and the $\mathrm{z}$-axis. Naturally, a quartz crystal has three $\mathrm{x}$-axes and three y-axes. Piezoelectric elements can be cut from a quartz crystal perpendicular to each of the three axes, as well as in some other planes. Moreover, in each case, piezoelectric elements have certain specific properties. The most commonly used piezoplates $x$ cut and at cut [6, 15-19]. The deformation of the piezoplates $\mathrm{x}$ and $\mathrm{y}$ cut is illustrated in [10-15]. In addition to these sections, piezoelectric elements of the 450 cut are used, with the help of which it is possible to excite shear vibrations in solids.

Segnet's salt (double potassium-sodium salt of tartaric acid). Easily soluble in water, it melts at a temperature of 58 ${ }^{\circ} \mathrm{C}$, and at a temperature of $54{ }^{\circ} \mathrm{C}$ it loses its piezoelectric properties. Piezoelectric and elastic constants of segnet's salt depend on temperature and can be considered approximately constant only in the range from 0 to $16{ }^{\circ} \mathrm{C}$. The main advantage of segnet's salt is its high piezoelectric effect, which is important in the development of highly sensitive acoustic receivers. The sensitivity of the segnet's salt receiver is about 100 times greater than the sensitivity of the quartz one, all other conditions being the same. Therefore, segnet's salt crystals are mainly used in the manufacture of piezoelectric elements for measuring equipment. In particular, they are widely used as ultrasonic flaw detection receivers. If an electric field $\mathrm{E}$ is created in such a piezoelectric element, a mechanical stress arises equal to:

$$
\sigma=e_{i j} E
$$

where, $\mathrm{e}_{\mathrm{ij}}-$ piezoelectric constant;

$$
e_{i j}=\frac{1}{2} d_{i j} E_{Y u}
$$

where, $d_{i j}$ - piezomodule; $E_{Y u}-$ Young's modulus.

Piezoceramics. At present, the following main types of ceramics have become widespread for the manufacture of piezoceramic elements: ceramics of barium titanate and its derivatives, ceramics of the zirconate-lead titanate (PZT) system and ceramics based on barium niobate lead. Piezoceramics of barium titanate $\left(\mathrm{BaJiO}_{3}\right)$ are characterized by a lower piezoelectric effect in comparison with Rochelle salt, 
Table 1. Physical and mechanical characteristics of piezomaterials

\begin{tabular}{|c|c|c|c|c|c|c|c|c|c|c|c|}
\hline 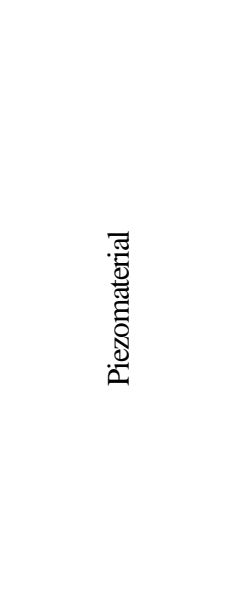 & 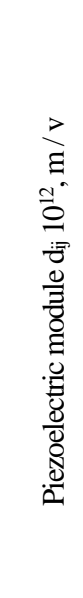 & 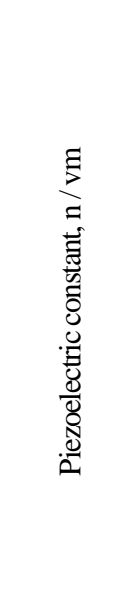 & 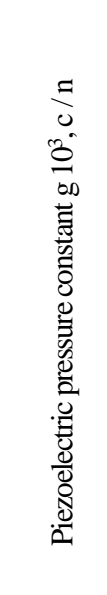 & 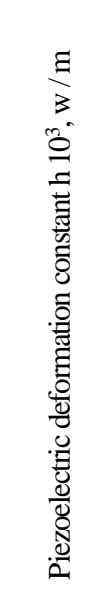 & 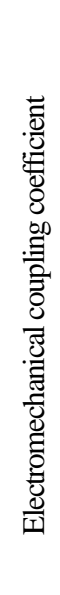 & 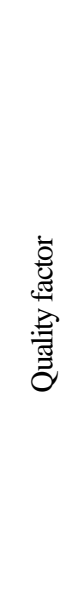 & 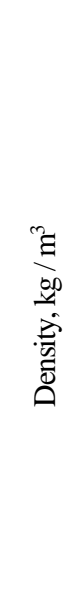 & 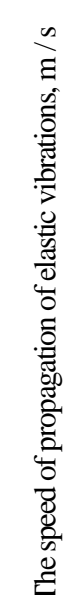 & 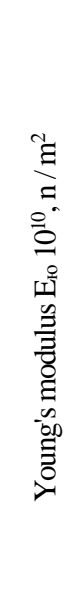 & 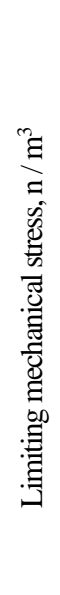 & 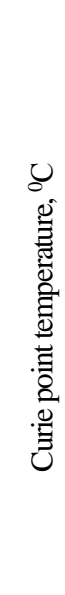 \\
\hline Quartz & 2,3 & 0,17 & 57 & 4,8 & 10 & 106 & 2,65 & 5,74 & 8,6 & 95 & 550 \\
\hline Segnet's salt & $\begin{array}{l}36 / 25 \\
11 / 55 \\
\end{array}$ & $\begin{array}{c}36 / 25 \\
0,11 / 0,08 \\
\end{array}$ & $\begin{array}{c}36 / 25 \\
160 / 600 \\
\end{array}$ & $\begin{array}{c}36 / 25 \\
1,6 / 1,9 \\
\end{array}$ & 29 & 103 & 1,77 & 2,4 & 1,0 & 14 & 45 \\
\hline Lithium sulfate & 16 & 0,9 & 190 & 9 & 38 & 103 & 2,06 & 5,46 & 6,2 & 15 & 75 \\
\hline Barium titanate & 150 & 16,7 & 12 & 1,2 & 50 & 400 & 5,5 & 5,68 & 18,0 & 80 & 100 \\
\hline $\begin{array}{c}\text { Lead zirconate } \\
\text { titanate }\end{array}$ & 33 & 16,7 & 33 & 1,7 & 50 & 400 & 7,0 & 5,0 & 20,0 & 80 & 350 \\
\hline Lead metaniobate & 165 & 4,8 & 40 & 1,2 & 42 & 11 & 5,8 & 2,76 & 0,46 & - & 550 \\
\hline
\end{tabular}

but greater in comparison with the piezoelectric effect of quartz. Barium titanate is insoluble in water, the second order phase transition (Curie point) corresponds to a temperature of $120{ }^{\circ} \mathrm{C}$. Piezoelectric elements made of barium titanate ceramics with the addition of 4-8\% (PbJiO3) lead titanate can operate in the temperature range up to $+150{ }^{\circ} \mathrm{C}$. Currently known piezoelectric ceramics of barium lead niobate, the Curie point of which corresponds to $2600 \mathrm{C}$. In terms of elastic properties, piezoelectric barium titanate is closest to quartz. The dependence of the fundamental natural vibration frequency on the thickness of a piezoelectric element made of barium titanate is given in $[6,16]$. One of the advantages of barium titanate is a high conversion rate of electrical energy into mechanical vibration energy. Particularly difficult operating conditions of the transducers at high mechanical stresses and in strong electric fields predetermined the use of barium titanate modified with calcium and cobalt, a material that is difficult to manufacture, possessing not very high indicators of piezoelectric properties, but relatively more stable when operating under these conditions. The $\mathrm{Pb}$ $\left(\mathrm{Zr}_{0.55} \mathrm{Ji}_{0.45}\right) \mathrm{O}_{3}$ piezoelectric ceramic PZT is characterized by a piezoelectric effect that is twice as large as compared to barium titanium. It is insoluble in water and has a Curie point of $330^{\circ} \mathrm{C}$. The strength of the PZT ceramics is greater than the strength of the barium titanate ceramics. Therefore, recently, PZT ceramics have been used mainly for work in difficult technological conditions. Billets from PZT piezoceramics, as well as from barium titanate ceramics, allow mechanical processing (cutting, grinding, polishing) to obtain piezoelectric elements of specified shapes and sizes. Currently, piezoceramics has become more widespread in ultrasonic and sound technology due to its relatively low cost, the possibility of manufacturing piezoelectric elements of various shapes from it, creating focusing systems, etc. Moreover, it can be made to emit and receive elastic vibrations with equal efficiency in any direction. These and other advantages of piezoelectric ceramics are the reason for the great attention that is paid to the development and improvement of piezoceramics in order to improve the elastic-strength and piezoelectric properties.

\section{Electrical model of piezo element}

A piezoelectric element is an electromechanical system, then to simplify the calculation of its parameters, an analogy can be drawn between the mechanical and electrical characteristics of the material. To do this, compare the differential equations, compiled according to the second Kirchhoff's law for a sequential $R-L-C$ circuit, and a mechanical oscillatory system. The first equation is:

$$
L \frac{d i(t)}{d t}+R i(t)+\frac{1}{C} \int i(t) d t=e(t)
$$

where $L, R, C$ - electrical inductance, resistance and capacitance of the series oscillatory circuit; $e(t)$ Electromotive force (EMF) of the source connected to the circuit, and EMF can be expressed in terms of voltage: $e(t)=$ $u(t)$. The equation for a mechanical vibrational system can be written as:

$$
m \frac{d^{2} x(t)}{d t^{2}}+C \frac{d x(t)}{d t}+k x(t)=F(t)
$$

Where $m, c, k$ - mass, damping coefficient and elasticity coefficient, and, $k=1 / \lambda, \lambda$ - compliance; $\mathrm{x}$ is the displacement under the action of the force F. Taking into account that the time derivative of displacement is the 
velocity $v(t)=d x(t) / d t$, the equation can be rewritten as:

$$
m \frac{d v(t)}{d t}+c v(t)+\frac{1}{\lambda} \int v(t) d t=F(t)
$$

Thus, it is possible to draw analogies between the electrical and mechanical characteristics of the system: Voltage - $u$; strength - $F$; electric current - $i$; speed $-v$; inductance - $L$; mass - $m$; electrical resistance - $R$; damping factor - $c$; capacity - $C$; compliance - $\lambda$. Consequently, the inductive resistance of the electrical system must be equal to the inertial resistance of the mechanical one, and the capacitive one - to the elastic one:

$$
\begin{aligned}
& \omega L=\omega m=z_{\text {ин }} \\
& \frac{1}{\omega C}=\frac{1}{\omega \lambda}=z_{\text {упр }}
\end{aligned}
$$

The electrical impedance must be equal to the mechanical:

$$
Z_{\ni}=\frac{u}{i}=\frac{F}{v}=Z_{M}
$$

Taking into account the fact that a piezoelectric element from an electrical point of view is a capacitor, then, using the analogies given above, it will be correct to model the electromechanical system of a piezoelectric element in the form of an electric circuit shown in fig. 2.

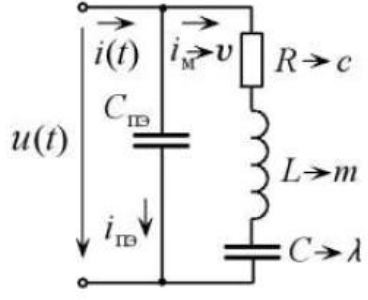

a

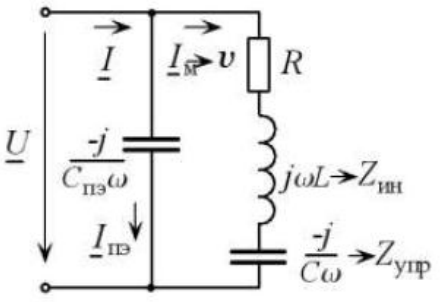

Fig. 2. Electric model of a piezoelectric element: a - in temporary form; $\mathrm{b}$ - in complex form

Scheme fig. 2 is a complex oscillatory circuit in which the resonance of currents in parallel branches and resonance of voltages (mechanical resonance or antiresonance) in a series oscillatory circuit can be observed.

\section{Practical research on the selection of the optimal structure of piezo ceramics}

The proposed piezoelectric transducer with a magnetoacoustic material layer was used as emitters for the extractor. Note that in the problems of ultrasonic tomography, predominantly longitudinal waves are used, for which the dependence of the velocity in a magnetoacoustic material on the field is much weaker. Nevertheless, here, too, the external magnetic field makes it possible to control the characteristics of the converter in a wide range. In fig. 3 shows the amplitude - frequency characteristic of the transducer in the prima mode for different magnetic fields, which made it possible to control the resonant frequency of the reception of the transducer developed by us up to $20 \%$, and its $\mathrm{Q}$ factor (and, accordingly, the operating frequency band) - up to $70 \%$.

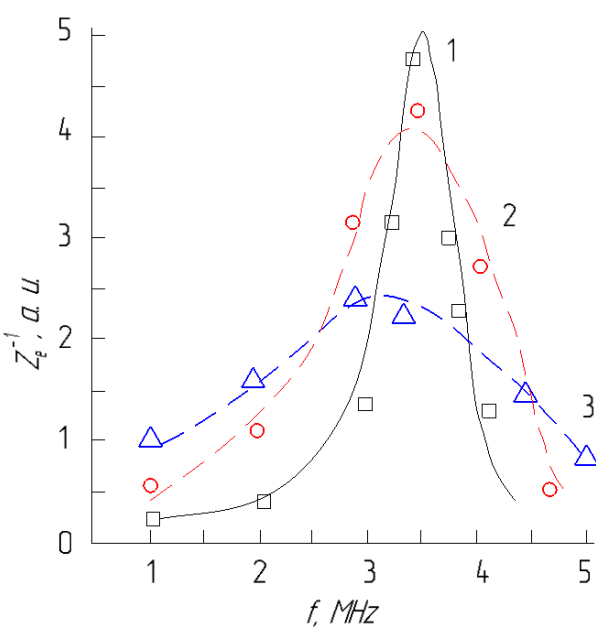

Fig. 3. Amplitude - frequency response of the longitudinal wave transducer loaded on water. $1-\mathrm{H}=2000 \mathrm{Oe}, 2-\mathrm{H}=500 \mathrm{Oe}, 3$ - $\mathrm{H}=100$ Oe.

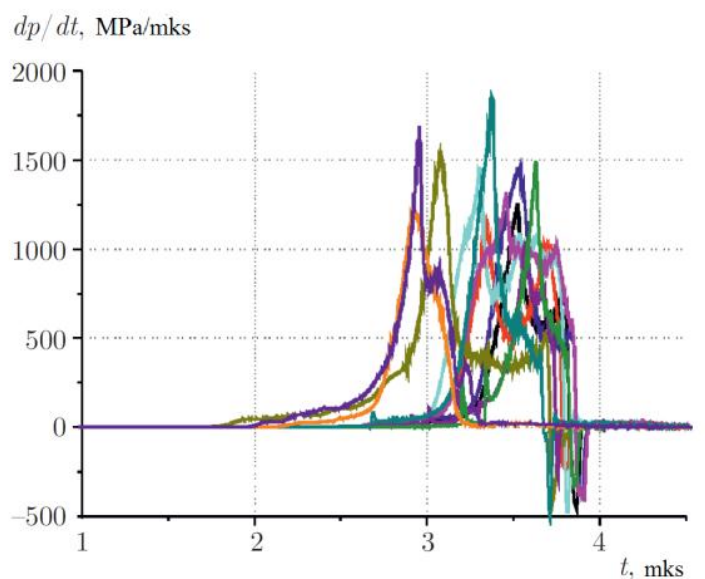

Fig. 4. Dependences of the rate of pressure rise recorded by piezoceramic sensors installed at a distance of $10 \mathrm{~mm}$ from the sample, which show signs of electrical breakdown

For piezoceramic sensors with a sharp drop in signals, see fig. 4 , the calculation of the electric fields that arose before the breakdown, made according to formula (10), gave the values $E_{\max }=1.7-3.3 \mathrm{kV} / \mathrm{mm}$, close to the value of the electric strength $(4 \mathrm{kV} / \mathrm{mm})$ for piezoelectric ceramics PZT -21 [7]. The presence of such fields makes it possible to explain the onset of breakdown, which caused the decay of signals in the experiments..

$$
\left|E_{\max }\right|=\frac{d_{i j} k x_{0}}{2 \varepsilon \varepsilon_{0}}
$$

It should be noted that the calculation of the electric field in the piezoelectric element, made without taking into account the field inhomogeneity arising at a high rate of pressure rise, gives $E=200 \mathrm{~V} / 0.5 \mathrm{~mm}=0.4 \mathrm{kV} / \mathrm{mm}$. When calculating using formula (10), the following parameters were used for PZT $-21: \varepsilon=480, c=3.73 \mathrm{~mm} / \mathrm{mks}, d_{i j}=110 \mathrm{pC} / \mathrm{n}$ and the pressure rise rate at the peaks $\mathrm{dp} / \mathrm{dt}_{\max }=1000-1900$ $\mathrm{MPa} / \mathrm{mks}$... 


\section{Conclusions}

The proposed scheme for solving the problem uses the method of successive approximations, which allows obtaining analytical expressions for calculating the coefficients in the mathematical description of the electric field potential in the volume of a disk with an anisotropic dielectric constant.

The piezoelectric effect is widely used today in various electromechanical devices and systems. Therefore, the predictability of the characteristics of piezoelectric elements in the production of such systems is the main design task. The proposed algorithm for calculating the frequency characteristics of a piezoelectric element makes it possible, on the basis of the data provided by the manufacturer of the piezoelectric ceramics, to determine such parameters of a piezoelectric element of a given shape as its resonant and antiresonant frequencies, bandwidth and cutoff frequencies of the passband. The calculation of these parameters is simple, with its help it is possible to construct a family of frequency characteristics for various dimensions of a piezoelectric element, which makes it possible for developers of electromechanical systems based on the piezoelectric effect to determine the optimal design of a sensitive or actuating element.

\section{References}

1. V. Sharapov, Zh. Sotula, L. Kunitskaya, Piezoelectric electroacoustic transducers. Heidelberg, Dordrecht, London, New York, Springer Verlag, 240 p. (2013).

2. V. M. Sharapov, I. G. Minaev, Zh. V. Sotula, L. G. Kunickaya E'lektroakusticheskie preobrazovateli. Moscow, Texnosfera, 280 p. (2013).

3. N. S. Koshlyakov, E'. B. Gliner, M. M. Smirnov, Uravneniya $v$ chastny'x proizvodny'x matematicheskoj fiziki. Moscow, Vy'sshaya shkola, 712 p. (1970).

4. E.P. Osadchiy, ed. Proektirovanie datchikov dlya izmereniya mekhanicheskikh velichin [Design of sensors for the measurement of mechanical values]. Moscow, Mashinostroenie Publ., 480 p. (1979).

5. K. Uchino, Entrepreneurship for engineers. New York, NY: CRC Press; (2009).

6. K. Uchino, Ferroelectric devices. 2nd ed. New York, NY: CRC Press; (2009).

7. V Bharti, HS Xu, G Shanti, QM Zhang, K Liang. Polarization and structural properties of high-energy electron irradiated poly(vinylidene fluoridetrifluoroethylene) copolymer films. J Appl Phys 2000;87:452. 8. Y Saito, H Takao, T Tani, T Nonoyama, K Takatori, T Homma, et al. Lead-free piezoceramics. Nature;432:84. (2004).

9. K. Uchino. Micromechatronics. New York, NY: CRC/Dekker; (2003).

10. K. Uchino. Ferroelectric devices \& piezoelectric actuators. Lancaster, PA: DEStech Pub; (2016).

11. H Takeuchi, H Masuzawa, C Nakaya, Y Ito. In: Proc. IEEE 1990 ultrasonics symposium; p. 697. (1990)

12. WA Smith. In: Proc. 1989 IEEE ultrasonic symposium; p. 755. (1989)

13. S Kalpat, X Du, IR Abothu, A Akiba, H Goto, K Uchino. Effect of crystal orientation on dielectric properties of lead zirconate titanate thin films prepared by reactive $R F$ sputtering. Jpn J Appl Phys;40:713. (2001).

14. I.U. Rakhmonov, K.M. Reymov, S.H. Dustova Improvements in industrial energy rationing methods. IOP Conference Series: Materials Science and Engineering. 862(6). P.1-5. (2020).

15. I. Rakhmonov, A. Berdishev, N. Niyozov, A. Muratov, U. Khaliknazarov. Development of a scheme for generating the predicted value of specific electricity consumption IOP Conference Series: Materials Science and Engineering. 883(1). P.1-8. (2020).

16. Sh.Sultanova, J. Safarov, A. Usenov, T. Raxmanova Definitions of useful energy and temperature at the outlet of solar collectors E3S Web of Conferences: Rudenko International Conference "Methodological problems in reliability study of large energy systems" 216, P.1-5. (2020).

17. Oumayma Boukria, El Mestafa El Hadrami, Shaxnoza Sultanova, Jasur Safarov, Francoise Leriche, Abderrahmane Ait-Kaddour 2D-Cross Correlation Spectroscopy Coupled with Molecular Fluorescence Spectroscopy for Analysis of Molecular Structure Modification of Camel Milk and Cow Milk Mixtures during Coagulation. Foods, 9(6), 724, P.1-10. (2020).

18. J.E. Safarov, Sh.A. Sultanova, G.T. Dadayev, Sh.U. Zulpanov Influence of the structure of coolant flows on the temperature profile by phases in a water heating dryer. IOP Conf. Series: Materials Science and Engineering. Dynamics of Technical Systems (DTS 2020). 1029, 012019. P.1-11. (2021).

19. J. Safarov, A. Khujakulov, Sh. Sultanova, U. Khujakulov, Sunil Verma Research on energy efficient kinetics of drying raw material. E3S Web of Conferences: Rudenko International Conference "Methodological problems in reliability study of large energy systems" (RSES 2020). 216. P.1-5. (2021). 